\title{
A Practical Guide for Using Myelofibrosis Prognostic Models in the Clinic
}

\author{
Joan How, MD, 1,2,3 and Gabriela S. Hobbs, MD
}

\section{ABSTRACT}

Primary myelofibrosis (PMF) has the least favorable prognosis of the Philadelphia chromosome-negative myeloproliferative neoplasms, which also include essential thrombocythemia (ET) and polycythemia vera (PV). However, clinical presentations and outcomes of PMF vary widely, with median overall survival ranging from years to decades. Given the heterogeneity of PMF, there has been considerable effort to develop discriminatory prognostic models to help with management decisions, particularly for the consideration of hematopoietic stem cell transplantation in patients at higher risk. Although earlier models incorporated only clinical features in risk stratification, contemporary models increasingly use molecular and cytogenetic features, leading to more comprehensive prognostication. This article reviews the most widely adopted prognostic models used for PMF, including the International Prognostic Scoring System (IPSS), dynamic IPSS (DIPSS)/DIPSS+, mutation-enhanced IPSS for transplant-age patients (MIPSS70)/MIPSS70+/MIPSS70+ version 2.0, genetically inspired prognostic scoring system, and Myelofibrosis Secondary to PV and ET-Prognostic Model in patients with post-ET/PV myelofibrosis. We also discuss newly emerging prognostic models and provide a practical approach to risk stratification in patients with PMF and post-ET/PV myelofibrosis.

J Natl Compr Canc Netw 2020;18(9):1271-1278 doi: $10.6004 /$ jnccn.2020.7557

${ }^{1}$ Department of Medical Oncology, Massachusetts General Hospital, Harvard Medical School; 'Division of Hematology, Department of Medicine, Brigham and Women's Hospital, Harvard Medical School; and ${ }^{3}$ Department of Medical Oncology, Dana-Farber Cancer Institute, Harvard Medical School, Boston, Massachusetts.
Primary myelofibrosis (PMF) is a myeloproliferative neoplasm (MPN) characterized by clonal hematopoietic cell expansion with bone marrow fibrosis. PMF has the worst prognosis of the classic Philadelphia chromosomenegative MPNs, with median overall survival (OS) estimated at 6 years. ${ }^{1}$ Although the most frequent cause of death is hematologic transformation and disease progression, patients with PMF are at high risk for other competing causes of morbidity and mortality. ${ }^{2}$ The considerable heterogeneity of PMF translates into a wide range of outcomes, with OS spanning a few years to decades. Given this heterogeneity, risk stratification for individualized management becomes essential in disease treatment. This review describes the development and major features of prognostic models currently available for use in PMF and provides a practical guide for how to approach risk stratification.

\section{Historical Prognostic Models}

The earliest prognostic studies in PMF were limited by the relative rarity of the disease, resulting in smaller sample sizes of 100 to 200 patients. ${ }^{3-7}$ The largest study at the time by Dupriez et $\mathrm{al}^{3}$ evaluated 195 patients with PMF and resulted in the most widely adopted early prognostic model, the Lille score. This simple scoring system used only 2 covariates: a hemoglobin (Hgb) level $<10 \mathrm{~g} / \mathrm{dL}$ and a WBC count $<4 \times 10^{9} / \mathrm{L}$ or $>30 \times 10^{9} / \mathrm{L}^{3}$ With the presence or absence of each variable at diagnosis, 3 risk groups were identified (low $=$ no risk factors; intermediate $=$ either low Hgb or low/high WBC; high = both low Hgb and low/high WBC), with median OS estimated at 93, 26, and 13 months, respectively. However, the Lille score did not separate patients at intermediate risk from those at high risk well. ${ }^{2}$ Efforts to improve on the Lille score included the addition of other risk factors, including thrombocytopenia and monocytosis. ${ }^{8}$ Further refinement of these earlier models required multicenter cooperation to acquire the larger sample sizes needed for more accurate risk stratification, resulting in the development of current contemporary models (Table 1).

\section{Contemporary Prognostic Models}

International Prognostic Scoring System

The purpose of the International Prognostic Scoring System (IPSS) was to provide prognostication and help 


\section{Table 1. Summary of Contemporary Prognostic Models for PMF and Post-ET/PV MF}

\section{Prognostic Model}

IPSS $^{2}$

DIPSS $+{ }^{15}$

MIPSS7020

MIPSS70 +20

MIPSS70+ v2.021

DIPSS $^{9}$

GIPSS $^{24}$
Variables (Scoring)

Risk Groups (Median or 5-y OS)

Age $>65$ y (1)

$\mathrm{Hgb}<10 \mathrm{~g} / \mathrm{dL}(1)$

WBC $>25 \times 10^{\circ} / \mathrm{L}(1)$

Circulating blasts $\geq 1 \%$ (1)

Constitutional symptoms (1)

Age $>65$ y (1)

$0=\operatorname{low}(11.3 \mathrm{y})$

$1=$ intermediate-1 $(7.9 \mathrm{y})$

2 = intermediate- $2(4.0 \mathrm{y})$

$\geq 3=\operatorname{high}(2.3 \mathrm{y})$

$\mathrm{Hgb}<10 \mathrm{~g} / \mathrm{dL}(2)$

$0=$ low (not reached)

WBC $>25 \times 10^{\circ} / \mathrm{L}(1)$

1-2 = intermediate-1 (14.2 y)

Circulating blasts $\geq 1 \%$ (1)

3-4 = intermediate-2 (4.0 y)

Constitutional symptoms (1)

\begin{tabular}{lrl} 
DIPSS low (0) & $0=$ low $(15.4 \mathrm{y})$ \\
\hline DIPSS int-1 (1) & $1=$ intermediate- $1(6.5 \mathrm{y})$ \\
\hline DIPSS int-2 (2) & $2-3=$ intermediate-2 $(2.9 \mathrm{y})$ \\
\hline DIPSS high (3) & $4-6=$ high $(1.3 \mathrm{y})$
\end{tabular}

Unfavorable karyotype (1)

Red cell transfusion need (1)

Platelets $<100 \times 10^{\circ} / \mathrm{L}(1)$

$\mathrm{Hgb}<10 \mathrm{~g} / \mathrm{dL}$ (1)

0-1 = low (27.7 y)

WBC $>25 \times 10^{9} / \mathrm{L}(2)$

2-4 = intermediate (7.1 y)

Circulating blasts $\geq 2 \%$ (1) $\geq 5=\operatorname{high}(2.3 \mathrm{y})$

Constitutional symptoms (1)

Platelets $<100 \times 10^{9} / \mathrm{L}(2)$

Bone marrow fibrosis grade $\geq 2$ (1)

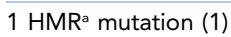

$\geq 2$ HMR $^{a}$ mutations (2)

Type 1/like CALR absent (1)

$\mathrm{Hgb}<10 \mathrm{~g} / \mathrm{dL}$ (1)

$0-2=$ low $(20 \mathrm{y})$

Circulating blasts $\geq 2 \%$ (1)

$3=$ intermediate $(6.3 \mathrm{y})$

Constitutional symptoms (1)

4-6 = high (3.9 y)

$1 \mathrm{HMR}^{\mathrm{a}}$ mutation (1)

$\geq 7=\operatorname{very}$ high $(1.7 \mathrm{y})$

$\geq 2$ HMR $^{a}$ mutations (2)

Type 1/like CALR absent (2)

Unfavorable karyotype ${ }^{\text {a }}$ (3)

VHR karyotype (2)

$0=\operatorname{low}(26.4 \mathrm{y})$

Unfavorable karyotype (1)

$1=$ intermediate- 1 ( $8.0 \mathrm{y})$

Type 1/like CALR absent (1)

2 = intermediate- $2(4.2 \mathrm{y})$

ASXL1 (1) $\geq 3=$ high $(2.0 \mathrm{y})$

SRSF2 (1)

U2AF1Q157 (1)

VHR karyotype (4)

$0=$ very low (not reached)

Unfavorable karyotype (3)

$1-2=\operatorname{low}(16.4 \mathrm{y})$

$\geq 2 \mathrm{HMR}^{\mathrm{a}}$ mutations (3)

3-4 = intermediate (7.7 y)

$1 \mathrm{HMR}^{\mathrm{a}}$ mutation (2)

5-8 = high (4.1 y)

Type 1/like CALR absent (2)

$\geq 9=$ very high $(1.8 \mathrm{y})$

Severe anemiab (2)

Moderate anemia ${ }^{c}(1)$

Circulating blasts $\geq 2 \%$ (1)

Constitutional symptoms (2) 


\section{Table 1. Summary of Contemporary Prognostic Models for PMF and Post-ET/PV MF (cont.)}

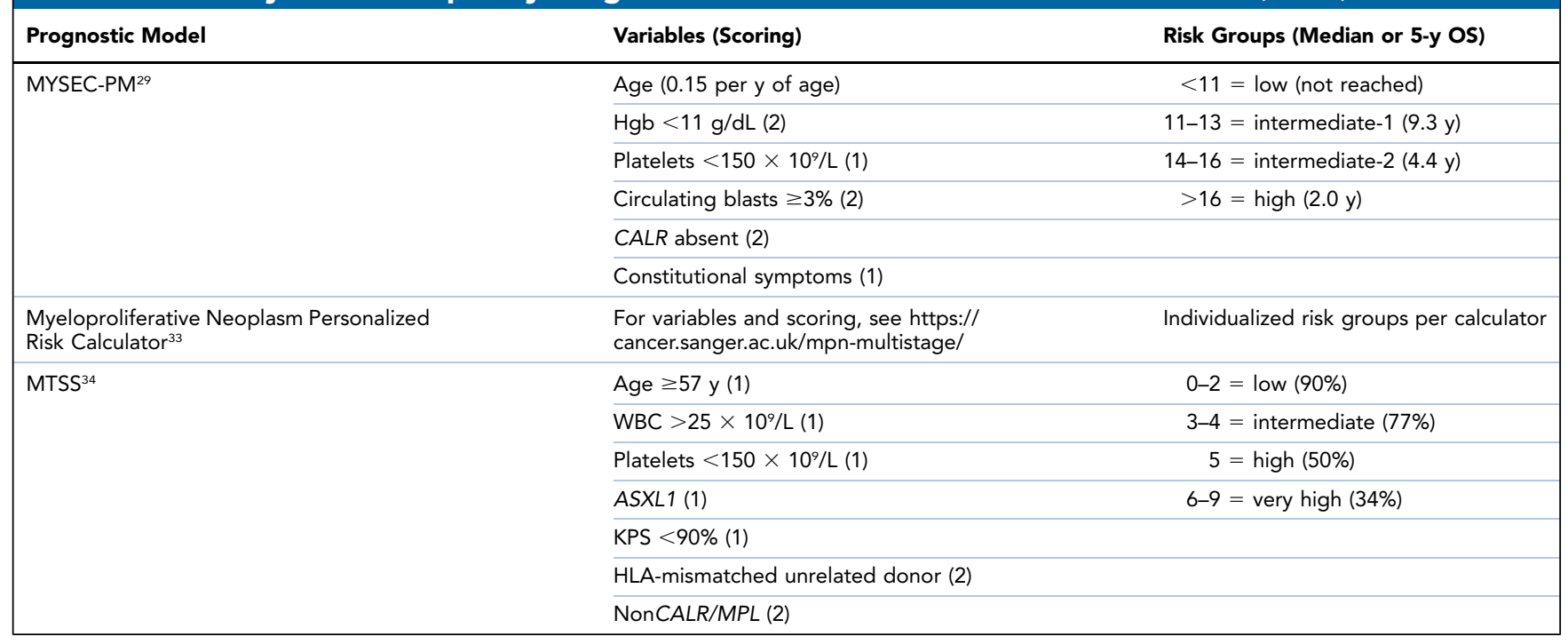

Abbreviations: DIPSS, dynamic IPSS; DIPSS+, dynamic IPSS plus; ET, essential thrombocythemia; GIPSS, genetically inspired prognostic scoring system; Hgb, hemoglobin; HLA, human leukocyte antigen; HMR, high molecular risk; IPSS, International Prognostic Scoring System; KPS, Karnofsky performance status; MF, myelofibrosis; MIPSS70, mutation-enhanced prognostic scoring system for transplant-age patients; MIPSS70+, mutation-enhanced prognostic scoring system for transplant-age patients plus; MIPSS70+ v2.0, mutation-enhanced prognostic scoring system for transplant-age patients plus version 2.0; MTSS, Myelofibrosis Transplant Scoring System; MYSEC-PM, Myelofibrosis Secondary to PV and ET-Prognostic Model; OS, overall survival; PMF, primary myelofibrosis; PV, polycythemia vera; VHR, very high risk.

aHMR mutation means the presence of a mutation in any of the following: ASXL1, SRSF2, EZH2, IDH1, IDH2; in addition, it means the presence of U2AF1Q157 in the GIPSS and MIPSS70+ v2.0.

bSevere anemia: Hgb level $<8 \mathrm{~g} / \mathrm{dL}$ in women and $<9 \mathrm{~g} / \mathrm{dL}$ in men

cModerate anemia: Hgb level of 8 to $9.9 \mathrm{~g} / \mathrm{dL}$ in women and 9 to $10.9 \mathrm{~g} / \mathrm{dL}$ in men.

with initial treatment decisions. ${ }^{2}$ Researchers evaluated 1,054 patients with PMF from 7 participating centers to develop the first contemporary scoring system widely adopted for clinical use. All variables were obtained at initial diagnosis, and patients with post-essential thrombocythemia (ET)/polycythemia vera (PV) myelofibrosis (MF) were excluded. The IPSS consisted of 5 variables: age $>65$ years, Hgb level $<10 \mathrm{~g} / \mathrm{dL}$, WBC count $>25 \times 10^{9} / \mathrm{L}$, circulating blast percentage $\geq 1 \%$, and presence of constitutional symptoms. With the presence of $0,1,2$, or $\geq 3$ variables, patients were divided into low-, intermediate-1, intermediate-2, and high-risk categories, with a median OS of $11.3,7.9,4.0$, and 2.3 years, respectively. Unlike in earlier models, the presence of leukopenia and a high monocyte percentage could not be validated. Cytogenetic variables were not included, and the IPSS was found to have higher discriminating power compared with earlier models, including the Lille score. ${ }^{2}$

\section{Dynamic IPSS and Dynamic IPSS+}

A major limitation of the IPSS was that all variables were obtained at diagnosis. Evaluation of risk factor acquisition would allow the development of a prognostic model that could be used during any disease time point, resulting in the conception of the dynamic IPSS (DIPSS). ${ }^{9}$ Passamonti et $\mathrm{al}^{9}$ evaluated 525 patients with PMF with regular follow-up and validated the acquisition of any IPSS risk factor as having a significant impact on OS. However, because anemia had a greater impact on prognosis compared with other risk factors, it was assigned 2 points for this score. The DIPSS divided patients into low-, intermediate-1, intermediate-2, and high-risk categories, corresponding to a median OS of not reached, 14.2 years, 4.0 years, and 1.5 years, respectively. The DIPSS was later found to predict leukemic progression in a separate analysis. ${ }^{10}$

Further IPSS-independent prognostic factors that impact OS in patients with PMF have been subsequently identified, including the need for red cell transfusion, ${ }^{11}$ unfavorable karyotype (Table 2), ${ }^{12,13}$ and thrombocytopenia. ${ }^{14}$ These features were added to the original variables of the DIPSS to develop the DIPSS.$+{ }^{15}$ Patients were similarly divided into low-, intermediate-1, intermediate-2, and high-risk groups with a respective median OS of 15.4, 6.5, 2.9, and 1.3 years. When evaluating prognostic factors for leukemia-free survival (LFS), only thrombocytopenia and unfavorable cytogenetics were identified as significant. ${ }^{15}$ Like the IPSS, both the DIPSS and the DIPSS + have been validated and adopted in official consensus guidelines for risk stratification in PMF $^{16,17}$ The DIPSS and DIPSS + remain easy-to-use prognostication tools today because they use readily 
available clinical variables and their dynamic nature allows the restaging of patients over time.

\section{Mutation-Enhanced IPSS}

Since the discovery of JAK2 mutations in 2005, understanding of the molecular biology of MPNs has increased exponentially. ${ }^{18}$ In addition to identification of driver mutations in CALR and MPL, a large number of other somatic mutations have been discovered that commonly occur in MPNs. ${ }^{19}$ Although the contribution of these genes to disease pathogenesis is being studied, certain mutations have been found to impact survival and transformation in MPNs. Inclusion of high-molecularrisk (HMR) mutations is a hallmark of the most recently developed scoring systems for PMF, which include the mutation-enhanced IPSS for transplant-age patients (MIPSS70, without cytogenetic information), ${ }^{20}$ the MIPSS70+ (with cytogenetic information), ${ }^{20}$ and, more recently, the MIPSS70+ version 2.0 (v2.0). ${ }^{21}$ These prognostic models were also significant for their inclusion of prefibrotic MF, which was a newly recognized WHO 2016 subclassification. ${ }^{22}$ In addition, the MIPSS70 was designed to help with transplantation decisions and therefore included only patients aged $<70$ years. However, both the MIPSS70 and the MIPSS70+ were validated in a separate PMF population that included patients aged $>70$ years. ${ }^{20}$

The MIPSS70 included both clinical risk factors (Hgb level $<10 \mathrm{~g} / \mathrm{dL}$, WBC count $>25 \times 10^{9} / \mathrm{L}$, platelet count $<100 \times 10^{9} / \mathrm{L}$, circulating blasts $\geq 2 \%$, bone marrow fibrosis grade $\geq 2$, presence of constitutional symptoms) and molecular risk factors (absence of CALR type 1/like mutation; presence of an HMR mutation, including ASXL1, SRSF2, EZH2, and IDH1/2; and $\geq 2$ HMR mutations). Unlike prior models, age was not included as a prognostic factor. However, the MIPSS70 was agerestricted for the express purpose of aiding transplant decision-making, recognizing that although age is consistently prognostic in PMF, it is often unhelpful in determining transplant eligibility given that older age itself is a relative contraindication to transplantation. The MIPSS70 model was a 3-tiered scoring system corresponding to a median OS of 27.7, 7.1, and 2.3 years, respectively. The MIPSS70+ included 3 clinical variables (Hgb level $<10 \mathrm{~g} / \mathrm{dL}$, circulating blasts $\geq 2 \%$, and constitutional symptoms) and incorporated the variable of an unfavorable karyotype (Table 2), resulting in a 4-tiered scoring system including a very-high-risk group (median OS, 1.7 years). Compared with the IPSS, risk redistribution in the MIPSS70 primarily consisted of upgrading patients from intermediate to high risk; however, the inferior OS of these patients suggested appropriate redistribution, validating the weight given to high-risk genetic variables in prognostication. The MIPSS70 was also found to be predictive of LFS.20
More recently, Tefferi et $\mathrm{al}^{21}$ published an updated MIPSS70+ v2.0 to incorporate newer genetic findings related to prognosis in PMF. Using the same patient population as the MIPSS70 and MIPSS70+, this latest scoring system differentiated patients with an unfavorable karyotype from those with a very-high-risk karyotype (Table 2$)^{23}$ and added U2AF1Q157 as an additional HMR mutation. ${ }^{24}$ The MIPSS70 + v2.0 also used a severity and sex-adjusted Hgb threshold for risk categorization (Table 1). The resulting 5 risk categories corresponded to a respective median OS of not reached, 16.4 years, 7.7 years, 4.1 years, and 1.8 years.

\section{Genetically Inspired Prognostic Scoring System}

The increasing amount of cytogenetic and molecular data emerging in PMF led to the development of a purely genetic scoring system, reflecting a hypothesis that all clinical variables in PMF are surrogates for known and unknown genetic markers. The genetically inspired prognostic scoring system (GIPSS) was designed as a simpler prognostic tool that would complement the more complex MIPSS70+ v2.0. ${ }^{25}$ The GIPSS evaluated 641 patients with PMF at diagnosis and was based on karyotype (very-high-risk, unfavorable, or favorable), absence of type 1/like CALR mutation, and presence of an HMR mutation (ASXL1, SRSF2, U2AF1Q157). Notably, EZH2 and $I D H 1 / 2$ mutations were not found to be significant in the GIPSS model. The GIPSS has 4 risk groups with 5-year OS of 26.4, 8.0, 4.2, and 2.0 years, respectively (Table 1). The GIPSS was found to be noninferior compared with the MIPSS70+ and the DIPSS and was validated for OS and LFS in a second cohort. $^{25}$

\section{Myelofibrosis Secondary to PV and ET-Prognostic Model}

All of the prognostic models discussed earlier evaluated patients with PMF only and specifically excluded those with post-ET/PV MF. Several studies have shown the decreased predictive power of the IPSS and DIPSS in patients with post-ET/PV MF, particularly when discriminating within intermediate-risk groups. ${ }^{26-28}$ This finding has led to an effort to develop a prognostic model specifically for post-ET/PV MF. Passamonti et $\mathrm{al}^{29}$ evaluated 685 patients with post-ET/PV MF at diagnosis and incorporated 6 variables associated with worse OS in the Myelofibrosis Secondary to PV and ET-Prognostic Model (MYSEC-PM): Hgb level $<11 \mathrm{~g} / \mathrm{dL}$, circulating blasts $\geq 3 \%$, lack of $C A L R$ mutation, platelet count $<150 \times 10^{9} / \mathrm{L}$, presence of constitutional symptoms, and older age. The MYSEC-PM is a 4-tiered scoring system with a median OS of not reached, 9.3 years, 4.4 years, and 2.0 years, respectively. It was validated in other post-ET/PV cohorts and found to be superior to the IPSS and DIPSS, 


\section{Table 2. Summary of Cytogenetic Risk Groups by Prognostic Model}

\begin{tabular}{|c|c|c|}
\hline & Unfavorable Karyotype & Very-High-Risk Karyotype \\
\hline DIPSS+ & $\begin{array}{l}\text { Presence of complex karyotype or sole or } 2 \text { abnormalities that } \\
\text { included }+8,-7 / 7 q-, i(17 q) \text {, inv(3), }-5 / 5 q-, 12 p-\text {, or } 11 q 23\end{array}$ & - \\
\hline MIPSS70+ & $\begin{array}{l}\text { Any karyotype other than normal karyotype or sole } \\
\text { abnormalities of } 20 q-, 13 q-,+9 \text {, chromosome } 1 \text { translocation/ } \\
\text { duplication, }-Y \text {, or sex chromosome abnormality other than }-Y\end{array}$ & 一 \\
\hline MIPSS70+ v2.0 & $\begin{array}{l}\text { Any karyotype other than very high-risk karyotype, normal } \\
\text { karyotype, or sole abnormalities of } 20 q_{-}, 13 q_{-}^{-},+9, \\
\text { chromosome } 1 \text { translocation/duplication, }-Y \text {, or sex } \\
\text { chromosome abnormality other than }-Y\end{array}$ & $\begin{array}{l}\text { Single or multiple abnormalities of }-7, \mathrm{i}(17 \mathrm{q}) \text {, inv }(3) / 3 q 21 \text {, } \\
12 p-/ 12 p 11.2,11 q-/ 11 q 23 \text {, or other autosomal trisomies not } \\
\text { including }+8 /+9\end{array}$ \\
\hline GIPSS & $\begin{array}{l}\text { Any karyotype other than very high-risk karyotype, normal } \\
\text { karyotype, or sole abnormalities of } 20 q^{-}, 13 q-,+9, \\
\text { chromosome } 1 \text { translocation/duplication, }-Y \text {, or sex } \\
\text { chromosome abnormality other than }-Y\end{array}$ & $\begin{array}{l}\text { Single or multiple abnormalities of }-7, i(17 q) \text {, inv }(3) / 3 q 21 \text {, } \\
12 p-/ 12 p 11.2,11 q-/ 11 q 23 \text {, or other autosomal trisomies not } \\
\text { including }+8 /+9\end{array}$ \\
\hline
\end{tabular}

Abbreviations: DIPSS +, dynamic international prognostic scoring system plus; GIPSS, genetically inspired prognostic scoring system; MIPSS70+, mutation-enhanced international prognostic scoring system for transplant-age patients plus; MIPSS70+ v2.0, mutation-enhanced international prognostic scoring system for transplantage patients plus version 2.0 .

with reclassification occurring primarily from higherto lower-risk categories. ${ }^{29-32}$

\section{Newly Emerging Models}

Grinfeld et $\mathrm{al}^{33}$ developed a personalized prognostic tool (https://cancer.sanger.ac.uk/mpn-multistage/) via wholeexome sequencing of 69 myeloid cancer genes in 2,000 patients with MPN, including 309 with PMF and postET/PV MF. This model allowed inputting of clinical and cytogenetic variables and individual mutations to predict patient-specific OS and risk of acute myeloid leukemia transformation. Unlike prior models, the MPN Personalized Risk Calculator recognized TP53 mutations as a particularly poor prognostic feature. Although this tool requires further experience and validation, it represents a novel approach to individualized risk stratification based primarily on genomic subgrouping.

In an effort to refine risk stratification specifically with regard to hematopoietic stem cell transplantation (HSCT) outcomes, Gagelmann et al ${ }^{34}$ recently published the Myelofibrosis Transplant Scoring System (MTSS). ${ }^{34}$ Using the MTSS, the authors evaluated 360 patients with PMF and post-ET/PV MF who underwent HSCT and identified prognostic variables specific for HSCT OS and nonrelapse mortality. Significant poor-risk variables included age $\geq 57$ years, Karnofsky performance status $<90 \%$, platelet count $<150 \times 10^{9} / \mathrm{L}$, WBC count $>25 \times 10^{9} / \mathrm{L}$, human leukocyte antigen-mismatched unrelated donor, ASXL1 mutation, and non-CALR/MPL driver mutation. Many variables with significant effect in the pretransplant setting (constitutional symptoms, cytogenetic risk stratification, Hgb level, $\geq 2$ HMR mutations) were not found to be prognostic in the MTSS. This finding suggests that HSCT can overcome some disease-related risk factors, allowing identification of patients who may particularly benefit from transplantation. The resulting 4-tiered scoring system (low, intermediate, high, very high) was more prognostic with regard to HSCT outcomes compared with the DIPSS, MIPSS70, and MYSEC-PM, ${ }^{34}$ although further experience is needed with this relatively new model.

\section{Practical Use of Prognostic Models}

Current consensus guidelines recommend use of the IPSS, DIPSS, and DIPSS + for risk stratification of PMF. ${ }^{16,17}$ Although NCCN recognizes the more recent molecularbased scoring systems in its guidelines and will likely include them in its next iteration, the official recommendations for risk stratification as of this writing are to use the more common and accessible IPSS, DIPSS, and DIPSS + scoring systems. ${ }^{16,17}$ In general, we favor using a risk stratification approach that incorporates molecular and cytogenetic information (ie, the MIPSS70+ v2.0), because prognostic models incorporating genetic features are more discriminative compared with models that incorporate clinical features alone. ${ }^{20,25}$ However, the DIPSS and DIPSS + are acceptable alternatives when molecular analysis and cytogenetics are not available.

In addition to providing prognostic information, the primary use for scoring systems is to determine which patients will benefit from HSCT. Current consensus guidelines recommend HSCT for patients at intermediate-2 and high risk using the IPSS, DIPSS, or DIPSS,$+{ }^{16,17}$ based on retrospective data showing superior OS in these patients when receiving HSCT versus nontransplant options. ${ }^{35}$ There are fewer data regarding the use of molecular-based scoring systems to aid transplant decision-making. The MIPSS70, MIPSS70+ v2.0, and GIPSS provide prognostic information with regard to transplant outcomes. ${ }^{34,36}$ In a more recent study applying the MIPSS70+ v2.0 to patients with MF undergoing 
reduced-intensity HSCT, Ali et $\mathrm{al}^{36}$ found a favorable 5 -year OS of $77 \%$ and $30 \%$ in patients at high and very high risk, respectively. These outcomes are significantly better than the approximate 5-year OS rates of $40 \%$ and $15 \%$ in patients at very high risk and high risk, respectively, reported previously by Teferri et al. ${ }^{21}$ The improved outcomes are likely affected by the strong selection bias seen in patients who ultimately are able to undergo HSCT. In addition, approximately half of the patients included in the more recent study by Ali et al ${ }^{36}$ had post-ET/PV MF, who were excluded in the development of the MIPSS70 + v2.0 and are not as clearly affected by HMR mutations as those with PMF. ${ }^{37}$ However, the encouraging outcomes reported after transplantation suggest a benefit in patients at high risk as stratified by the MIPSS70+ v2.0. In addition, given the abysmal prognosis associated with blastphase MPN, consideration of endpoints such as LFS may have more relevance in earlier transplant recommendations. Scoring systems that consider molecular and cytogenetic information, such as the MIPSS70 and GIPSS, also have increased prognostic value with specific regard to the risks of leukemia progression.

A simplified approach to risk stratification for HSCT consideration is illustrated in Figure 1. When mutation analysis and cytogenetics are available, the MIPSS70+ v2.0 is an effective tool for prognostication, with HSCT recommended for patients at very high risk and high risk. An alternative approach is to use the GIPSS as an initial screening tool to identify patients at high risk who would potentially benefit from HSCT, with further reevaluation using the MIPSS70+ v2.0 in patients at intermediate risk according to the GIPSS. The GIPSS is a simpler prognostic model that has an efficacy equivalent to the MIPSS70 + v2.0 in discriminating between patients at high risk and low risk ${ }^{25}$; however, the MIPSS70+ v2.0 is more effective in discriminating between patients at intermediate risk. The MIPSS70 can be used in patients for whom mutation analysis is available but cytogenetic information is not. The DIPSS and DIPSS + are acceptable alternatives if molecular information and cytogenetics are not obtainable. The MTSS can provide additional transplant-specific prognostication, although further experience is needed with this newer model.

The decision to undergo HSCT is complex, and we recommend early involvement of the bone marrow transplantation team when HSCT is being considered. Although improved understanding of clinical and genetic variables can more accurately inform which patients are likely to require HSCT during their disease course, the question of HSCT timing is not clear. For instance, immediate versus delayed HSCT may be options in younger patients who feel well and otherwise have lower risk based on clinical scores (ie, the DIPSS) but have poor-risk molecular scores (ie, the GIPSS), indicating a high probability of disease transformation and/or progression in the near future. In addition, the extent to which HSCT can overcome poor prognostic disease features is unclear, because patients at higher risk based on pretransplantation scoring also tend to do worse after HSCT, particularly because of increased nonrelapse mortality. ${ }^{38}$ For these patients, transplantation earlier in the disease course may be beneficial. Despite limitations
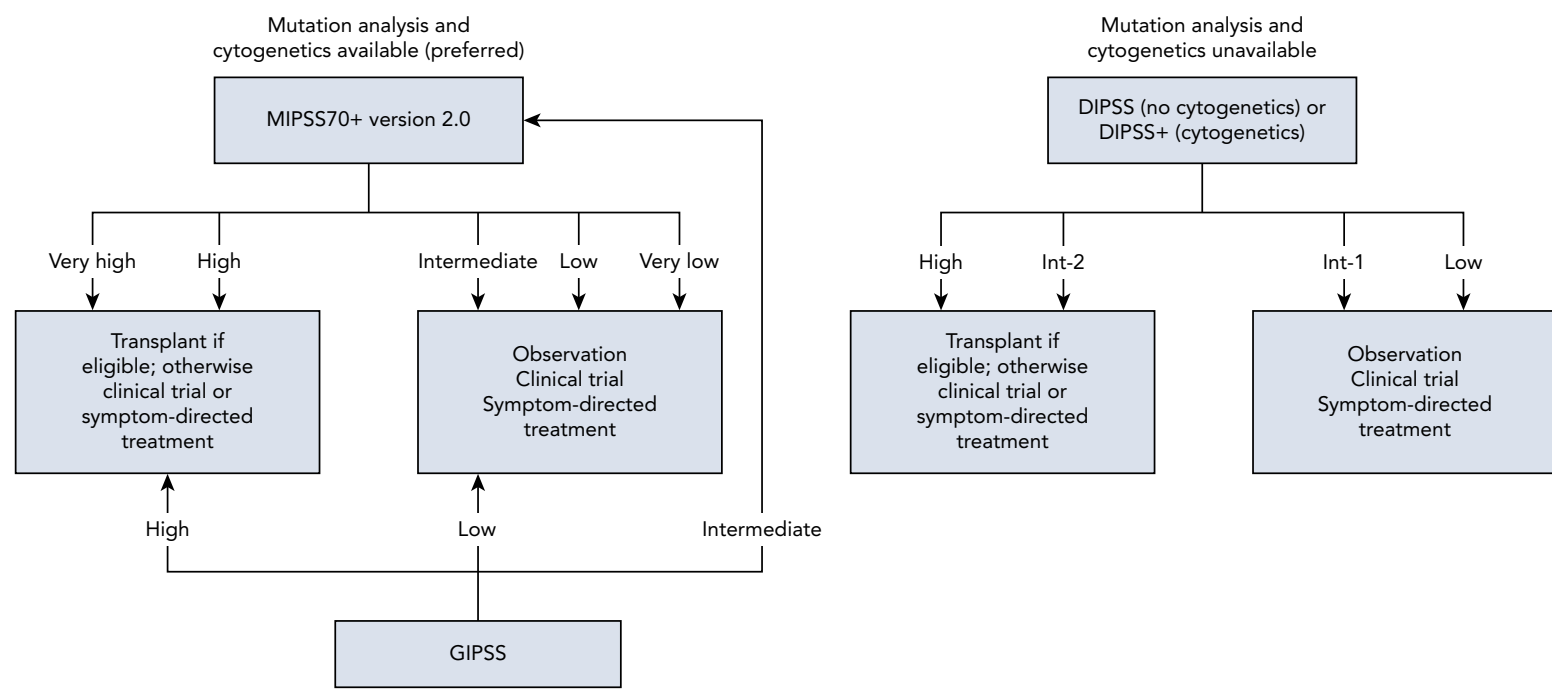

Figure 1. Approach to risk stratification in patients with primary myelofibrosis.

Abbreviations: DIPSS, dynamic international prognostic scoring system; DIPSS+, dynamic international prognostic scoring system plus; GIPSS, genetically inspired prognostic scoring system; Int-1, intermediate-1 risk; Int-2, intermediate-2 risk; MIPSS70+, mutation-enhanced international prognostic scoring system for transplantage patients plus. 
in determining the exact timing of HSCT, prognostic models are essential tools for initiating bone marrow transplantation referrals, which are critical in ensuring that patients receive appropriate counseling and guidance.

\section{Special Situations}

Post-ET/PV MF

As described previously, initial prognostic models specifically excluded patients with post-ET/PV MF, and current consensus guidelines make no distinction in the management or risk stratification of patients with PMF versus those with post-ET/PV MF. ${ }^{16,17}$ However, evidence shows that prognostic models for PMF have poorer performance in patients with post-ET/PV MF. ${ }^{26-28}$ In addition, molecular risk factors for post-ET/PV MF are not as well established as for PMF. Mutations in ASXL1, $E Z H 2$, and IDH1, which are considered HMR in PMF, have not been shown to be associated with worse outcomes in patients with post-ET/PV MF. ${ }^{37}$ This suggests that existing molecular models such as the MIPSS70 may be less applicable for post-ET/PV MF.

The MYSEC-PM was established specifically in patients with post-ET/PV $\mathrm{MF}^{29}$ and has shown superior predictive value compared with the IPSS and DIPSS in multiple subsequent studies. ${ }^{30-32}$ We recommend use of the MYSEC-PM for initial prognostication in patients with post-ET/PV MF at diagnosis, although its widespread adoption for risk stratification and transplant decision-making still requires further validation. Notably, age is calculated as a continuous variable in the MYSEC$\mathrm{PM}$ and contributes significantly to the overall prognostic score such that poorer-risk categories consist mostly of patients aged $>70$ years. This factor may reduce the number of patients eligible for transplant, and we recommend using information from other models, such as the DIPSS and DIPSS+, to help with management decisions.

\section{Prefibrotic MF}

In 2016, the WHO subclassified PMF into prefibrotic and overtly fibrotic categories, ${ }^{22}$ with the recognition that patients with prefibrotic MF have outcomes that are intermediate between those of patients with ET and overtly fibrotic PMF. ${ }^{39-42}$ Patients with prefibrotic MF were not included in the development and validation of the IPSS, DIPSS, and DIPSS + . When the IPSS was later analyzed in patients with prefibrotic MF, it could discriminate between low-, intermediate-, and high-risk groups; however, patients with intermediate- 1 and intermediate- 2 risk had a superimposable median OS $>10$ years. ${ }^{41}$ Therefore, unlike in patients with overt PMF, it would be premature to recommend HSCT in IPSS-categorized patients with intermediate-2 risk prefibrotic MF. However, patients with IPSS-categorized high-risk prefibrotic MF showed poor OS similar to their overt PMF counterparts, implying that HSCT could be considered for these patients.

The MIPSS70, MIPSS70+, and MIPSS70+ v2.0 included patients with prefibrotic MF in their training and validation cohorts. ${ }^{20,36}$ These prognostic models therefore have utility specifically in the prefibrotic MF population, and we recommend use of the MIPSS70+ v2.0 for patients with prefibrotic MF at diagnosis. In patients for whom molecular and/or cytogenetic information is unavailable, the IPSS, DIPSS, and DIPSS + can be useful for separating those at high risk who may benefit from more aggressive management approaches, including HSCT consideration.

\section{Conclusions and Future Directions}

The past decade has produced a wealth of new information regarding clinical and genetic risk factors in PMF, resulting in increasingly sophisticated prognostic models that help with patient management. As we gain further understanding of disease pathogenesis and biology, prognostic models are moving toward an emphasis on molecular and genetic risk stratification, which will likely make up the bulk of updates to existing scoring systems. Further validation of newer models in PMF and post-ET/PV MF is necessary, especially with regard to HSCT benefits and timing.

A major limitation to our current prognostic models is the relative scarcity of treatment options that exist for disease groups within PMF. Currently, risk stratification is critically important for consideration of HSCT in patients at high risk. However, in patients with PMF who are transplant-ineligible, management is similar between high- and low-risk groups, with the decision to initiate disease-directed therapy (eg, ruxolitinib, fedratinib, hydroxyurea) based primarily on the presence of symptoms. Ideally, prognostic models with molecular subtyping of disease will also inform specific therapy choices for different disease groups. More effective treatment strategies, including disease-modifying therapies, are necessary before the utility of prognostic models can truly be maximized.

Submitted December 23, 2019; accepted for publication March 4, 2020.

Disclosures: Dr. How has disclosed that she has no financial interests, arrangements, affiliations, or commercial interests with the manufacturers of any products discussed in this article or their competitors. Dr. Hobbs has disclosed that she receives grant/research support from Bayer, Incyte, and Merck; consulting fees from Incyte, Celgene, Agios, and Jazz Pharmaceuticals; and is a scientific advisor for Novartis, Celgene, Bristol-Myers Squibb, and Jazz Pharmaceuticals.

Correspondence: Gabriela Hobbs, MD, Massachusetts General Hospital, Department of Medical Oncology, Zero Emerson, Office 138, 55 Fruit Street, Boston, MA 02114. Email: ghobbs@partners.org 


\section{References}

1. Tefferi A, Guglielmelli P, Larson DR, et al. Long-term survival and blast transformation in molecularly annotated essential thrombocythemia, polycythemia vera, and myelofibrosis. Blood 2014;124:2507-2513.

2. Cervantes F, Dupriez B, Pereira A, et al. New prognostic scoring system for primary myelofibrosis based on a study of the International Working Group for Myelofibrosis Research and Treatment. Blood 2009;113: 2895-2901.

3. Dupriez B, Morel P, Demory JL, et al. Prognostic factors in agnogenic myeloid metaplasia: a report on 195 cases with a new scoring system. Blood 1996;88:1013-1018.

4. Cervantes F, Barosi G, Demory JL, et al. Myelofibrosis with myeloid metaplasia in young individuals: disease characteristics, prognostic factors and identification of risk groups. Br J Haematol 1998;102:684-690.

5. Cervantes F, Pereira A, Esteve J, et al. Identification of "short-lived" and "long-lived" patients at presentation of idiopathic myelofibrosis. Br J Haematol 1997:97:635-640.

6. Reilly JT, Snowden JA, Spearing RL, et al. Cytogenetic abnormalities and their prognostic significance in idiopathic myelofibrosis: a study of 106 cases. Br J Haematol 1997:98:96-102.

7. Kvasnicka HM, Thiele J, Werden C, et al. Prognostic factors in idiopathic (primary) osteomyelofibrosis. Cancer 1997;80:708-719.

8. Elliott MA, Verstovsek $S$, Dingli $D$, et al. Monocytosis is an adverse prognostic factor for survival in younger patients with primary myelofibrosis. Leuk Res 2007;31:1503-1509.

9. Passamonti F, Cervantes F, Vannucchi AM, et al. A dynamic prognostic model to predict survival in primary myelofibrosis: a study by the IWGMRT (International Working Group for Myeloproliferative Neoplasms Research and Treatment). Blood 2010;115:1703-1708.

10. Passamonti F, Cervantes F, Vannucchi AM, et al. Dynamic international prognostic scoring system (DIPSS) predicts progression to acute myeloid leukemia in primary myelofibrosis. Blood 2010;116:2857-2858.

11. Tefferi A, Siragusa S, Hussein K, et al. Transfusion-dependency at presentation and its acquisition in the first year of diagnosis are both equally detrimental for survival in primary myelofibrosis-prognostic relevance is independent of IPSS or karyotype. Am J Hematol 2010;85:14-17.

12. Hussein K, Pardanani AD, Van Dyke DL, et al. International Prognostic Scoring System-independent cytogenetic risk categorization in primary myelofibrosis. Blood 2010;115:496-499.

13. Caramazza D, Begna KH, Gangat N, et al. Refined cytogenetic-risk categorization for overall and leukemia-free survival in primary myelofibrosis: a single center study of 433 patients. Leukemia 2011;25:82-88.

14. Patnaik MM, Caramazza D, Gangat N, et al. Age and platelet count are IPSS-independent prognostic factors in young patients with primary myelofibrosis and complement IPSS in predicting very long or very short survival. Eur J Haematol 2010;84:105-108.

15. Gangat N, Caramazza D, Vaidya R, et al. DIPSS plus: a refined dynamic international prognostic scoring system for primary myelofibrosis that incorporates prognostic information from karyotype, platelet count, and transfusion status. J Clin Oncol 2011;29:392-397.

16. Gerds AT, Gotlib J, Bose P, et al. NCCN Clinical Practice Guidelines in Oncology: Myeloproliferative Neoplasms. Version 1.2020. Accessed July 27, 2020. To view the most recent version, visit NCCN.org

17. Kröger NM, Deeg JH, Olavarria $\mathrm{E}$, et al. Indication and management of allogeneic stem cell transplantation in primary myelofibrosis: a consensus process by an EBMT/ELN international working group. Leukemia 2015; 29:2126-2133.

18. Baxter EJ, Scott LM, Campbell PJ, et al. Acquired mutation of the tyrosine kinase JAK2 in human myeloproliferative disorders. Lancet 2005;365 1054-1061.

19. Vainchenker W, Delhommeau F, Constantinescu SN, et al. New mutations and pathogenesis of myeloproliferative neoplasms. Blood 2011;118: 1723-1735.

20. Guglielmelli P, Lasho TL, Rotunno G, et al. MIPSS70: mutation-enhanced international prognostic score system for transplantation-age patients with primary myelofibrosis. J Clin Oncol 2018;36:310-318.

21. Tefferi A, Guglielmelli P, Lasho TL, et al. MIPSS70+ version 2.0: mutation and karyotype-enhanced international prognostic scoring system for primary myelofibrosis. J Clin Oncol 2018;36:1769-1770.

22. Arber DA, Orazi A, Hasseriian R, et al. The 2016 revision to the World Health Organization classification of myeloid neoplasms and acute leukemia. Blood 2016;127:2391-2405.
23. Tefferi A, Nicolosi M, Mudireddy M, et al. Revised cytogenetic risk stratification in primary myelofibrosis: analysis based on 1002 informative patients. Leukemia 2018;32:1189-1199.

24. Tefferi A, Finke CM, Lasho TL, et al. U2AF1 mutation types in primary myelofibrosis: phenotypic and prognostic distinctions. Leukemia 2018;32 2274-2278.

25. Tefferi A, Guglielmelli P, Nicolosi M, et al. GIPSS: genetically inspired prognostic scoring system for primary myelofibrosis. Leukemia 2018;32 1631-1642.

26. Hernández-Boluda JC, Pereira A, Gómez M, et al. The International Prognostic Scoring System does not accurately discriminate different risk categories in patients with post-essential thrombocythemia and postpolycythemia vera myelofibrosis. Haematologica 2014;99:e55-57.

27. Masarova L, Bose P, Daver N, et al. Patients with post-essential thrombocythemia and post-polycythemia vera differ from patients with primary myelofibrosis. Leuk Res 2017:59:110-116.

28. Tefferi A, Saeed L, Hanson CA, et al. Application of current prognostic models for primary myelofibrosis in the setting of post-polycythemia vera or post-essential thrombocythemia myelofibrosis. Leukemia 2017;31: 2851-2852.

29. Passamonti F, Giorgino T, Mora B, et al. A clinical-molecular prognostic model to predict survival in patients with post polycythemia vera and post essential thrombocythemia myelofibrosis. Leukemia 2017:31:2726-2731.

30. Hernández-Boluda JC, Pereira A, Correa JG, et al. Performance of the myelofibrosis secondary to PV and ET-prognostic model (MYSEC-PM) in a series of 262 patients from the Spanish registry of myelofibrosis. Leukemia 2018;32:553-555.

31. Palandri $F$, Palumbo $G A$, lurlo $A$, et al. Differences in presenting features, outcome and prognostic models in patients with primary myelofibrosis and post-polycythemia vera and/or post-essential thrombocythemia myelofibrosis treated with ruxolitinib. New perspective of the MYSEC-PM in a large multicenter study. Semin Hemato 2018;55:248-255

32. Masarova L, Kantarjian H, Verstovsek S. Validation of the myelofibrosis secondary to PV and prognostic model in newly diagnosed patients with post-polycythemia vera and post-essential thrombocythemia myelofibrosis: MD Anderson Cancer Center. Clin Lymphoma Myeloma Leuk 2017;17(Suppl):S20-21.

33. Grinfeld J, Nangalia J, Baxter EJ, et al. Classification and personalized prognosis in myeloproliferative neoplasms. N Engl J Med 2018;379: 1416-1430.

34. Gagelmann N, Ditschkowski M, Bogdanov R, et al. Comprehensive clinical-molecular transplant scoring system for myelofibrosis undergoing stem cell transplantation. Blood 2019;133:2233-2242.

35. Kröger N, Giorgino T, Scott BL, et al. Impact of allogeneic stem cell transplantation on survival of patients less than 65 years of age with primary myelofibrosis. Blood 2015;125:3347-3350.

36. Ali H, Aldoss I, Yang D, et al. MIPSS70+ v2.0 predicts long-term survival in myelofibrosis after allogeneic $\mathrm{HCT}$ with the Flu/Mel conditioning regimen. Blood Adv 2019;3:83-95.

37. Rotunno G, Pacilli A, Artusi V, et al. Epidemiology and clinical relevance of mutations in postpolycythemia vera and postessential thrombocythemia myelofibrosis: a study on 359 patients of the AGIMM group. Am J Hematol 2016;91:681-686.

38. Scott BL, Gooley TA, Sorror ML, et al. The Dynamic International Prognostic Scoring System for myelofibrosis predicts outcomes after hematopoietic cell transplantation. Blood 2012;119:2657-2664.

39. Barbui T, Thiele J, Passamonti F, et al. Survival and disease progression in essential thrombocythemia are significantly influenced by accurate morphologic diagnosis: an international study. J Clin Oncol 2011;29: 3179-3184.

40. Rumi E, Boveri E, Bellini M, et al. Clinical course and outcome of essential thrombocythemia and prefibrotic myelofibrosis according to the revised WHO 2016 diagnostic criteria. Oncotarget 2017;8: 101735-101744.

41. Guglielmelli P, Pacilli A, Rotunno G, et al. Presentation and outcome of patients with $2016 \mathrm{WHO}$ diagnosis of prefibrotic and overt primary myelofibrosis. Blood 2017;129:3227-3236.

42. Mudireddy $M$, Shah $S$, Lasho $T$, et al. Prefibrotic versus overtly fibrotic primary myelofibrosis: clinical, cytogenetic, molecular and prognostic comparisons. Br J Haematol 2018;182:594-597. 